\title{
Expression of fibroblast growth factor receptor 2 IIIc in human uterine cervical intraepithelial neoplasia and cervical cancer
}

\author{
RIEKO KAWASE ${ }^{1,2}$, TOSHIYUKI ISHIWATA ${ }^{1}$, YOKO MATSUDA ${ }^{1}$, MUNEHIKO ONDA $^{1}$, \\ MITSUHIRO KUDO $^{1}$, TOSHIYUKI TAKESHITA ${ }^{2}$ and ZENYA NAITO ${ }^{1}$ \\ ${ }^{1}$ Department of Pathology, Integrative Oncological Pathology, Nippon Medical School, Tokyo 113-8602; \\ ${ }^{2}$ Division of Reproductive Medicine, Perinatology and Gynecologic Oncology, Graduate School \\ of Medicine, Nippon Medical School, Tokyo 113-8603, Japan
}

Received September 21, 2009; Accepted November 12, 2009

DOI: 10.3892/ijo_00000504

\begin{abstract}
Fibroblast growth factor receptors (FGFRs) 1-3 have $\mathrm{IIIb}$ and IIIc isoforms, and we reported that FGFR2 IIIb is highly expressed in cervical keratinizing squamous cell carcinoma (SCC). In this study, we determined the expression and roles of FGFR2 IIIc in cervical intraepithelial neoplasia (CIN) and cervical cancer. In CINs 1 and 2, FGFR2 IIIc was found to be localized at the basal to lower two-thirds of the squamous epithelium, whereas it was localized in most of the squamous epithelium, except for the superficial layer in CIN 3. In situ hybridization (ISH) analysis showed that the expression patterns of FGFR2 IIIc mRNA are similar to those of FGFR2 IIIc protein in CINs. The FGFR2 IIIc protein was detected in all invasive cervical cancer patients ( 29 cases) and its mRNA was found to be strongly expressed in the invasive front of cancer cell nests. FGFR2 IIIc cDNA was stably transfected into CaSki cells, which are derived from a cervical SCC. The growth rates of the CaSki cells were higher than those of Mock cells in vitro, and the CaSki cells tended to form larger subcutaneous tumors in nude mice. These findings suggest that FGFR2 IIIc plays important roles in carcinogenesis and growth of cervical cancer cells. Anti-FGFR2 IIIc therapies may represent therapeutic strategies for inhibiting the growth of CIN and cervical cancer.
\end{abstract}

\section{Introduction}

Cervical cancer is the second most common cancer among women worldwide and nearly one-third of patients who present with invasive cervical cancer die of this disease $(1,2)$. Human

Correspondence to: Dr Zenya Naito, Department of Pathology, Integrative Oncological Pathology, Nippon Medical School, 1-1-5 Sendagi, Bunkyo-ku, Tokyo 113-8602, Japan

E-mail: naito@nms.ac.jp

Key words: fibroblast growth factor receptor 2 IIIc/FGFR2 IIIc, cervical intraepithelial neoplasia/CIN, uterine cervical cancer, immunohistochemistry, in situ hybridization analysis papilloma virus (HPV) has been established as a causative agent of cervical carcinogenesis and the HPV DNA can be detected in $99 \%$ of patients with invasive squamous cell carcinoma (SCC) of the cervix (3). The major histological type of cervical cancer is SCC and cancer cells of this type were reported to synthesize many types of growth factor or growth factor receptors including the basic fibroblast growth factor (bFGF, FGF-2), FGF-7 (keratinocyte growth factor/ KGF), FGF receptor 3 (FGFR3), platelet-derived endothelial cell growth factor (PD-ECGF), vascular endothelial growth factor (VEGF)-A, VEGF-C, hepatocyte growth factor receptor (c-Met), insulin-like growth factor (IGF)-2, IGF-1 receptor (IGF-1R) and epidermal growth factor receptor (EGFR) (4-14). Serum levels of VEGF isoforms often increase in cervical cancer patients and decrease significantly following successful treatment (15-18).

The FGF family consists of 22 members, namely, aFGF (FGF-1), bFGF (FGF-2), int-2 (FGF-3), hst/K-FGF (FGF-4), FGF-5, FGF-6, FGF-7, androgen-induced growth factor (AIGF/FGF-8), glia-activating factor (GAF/FGF-9), FGF-10, FGF-11 (FGF homologous factor-3/FHF-3), FGF-12 (FHF-1), FGF-13 (FHF-2), FGF-14 (FHF-4) and FGF-16 to FGF-23 (19). FGFs bind to four high-affinity FGF receptors (FGFR1 to FGFR4) and low-affinity heparan sulfate proteoglycans that enhance ligand presentation (20). FGFRs are single transmembrane receptors, containing extracellular, transmembrane and intracellular domains (21). The extracellular domain of FGFRs is usually composed of three immunoglobulin (Ig)like domains, and the alternative splicing of the C-terminal half of the third Ig-like domain generates IIIb and IIIc isoforms in FGFRs 1-3 (22). IIIb isoforms are mainly localized in epithelial cells, but IIIc isoforms are mainly localized in mesenchymal cells in normal human tissues (23). Different FGFs bind to these two isoforms of FGFR with high affinity. FGFs-1, 3, 7, 10 and 22 bind to FGFR2 IIIb, whereas FGFs-1, 2, 4, 6, 8, 9, 17 and 18 strongly bind to FGFR2 IIIc $(19,21)$. The binding of different FGFs to FGFRs determines the mode of cell growth and cell behavior, including differentiation, migration and invasion.

The switching of the expression pattern of FGFR2 isoforms from IIIb to IIIc induces aggressive tumor growth and invasion in prostatic and bladder cancers $(24,25)$. Single nucleotide polymorphisms (SNPs) of the FGFR2 gene are associated 
with breast and endometrial cancers, and missense mutations or copy number gains of the FGFR2 gene occur in breast and gastric cancers $(22,26-28)$. Therefore, FGFR2 has been noted as a molecular target for cancer treatment in patients with FGFR2 mutation or gene amplification. In cervical cancer, cDNA array analysis showed higher FGFR2 expression in malignant cervical epithelial samples than in normal epithelial samples (29). Previously, we detected the expression of FGFR2 IIIb in $86 \%$ of cervical cancer patients, and this expression may mediate the growth and differentiation of reserve cells and SCC in the cervix (5). Furthermore, FGFs-1 and 2 are reported to be expressed in cervical cancer patients $(13,14)$. FGF-2 mRNA and its protein were also found to be highly expressed in advanced cervical cancer tissues, and the serum FGF-2 protein level was suggested to be a useful marker for the detection of primary tumors and recurrences, and monitoring of cancer therapy $(13,30)$. However, there has been no report on the expression and role of FGFR2 IIIc in cervical cancer. In this study, we examined whether FGFR2 IIIc mRNA and protein are expressed in cervical intraepithelial neoplasia (CIN) and invasive cervical cancer tissues. FGFR2 IIIc expression was also examined in cervical cancer cell lines.

We now report that the FGFR2 IIIc expression pattern is correlated with CIN progression. FGFR2 IIIc was expressed in all the tested cancer cell lines and SCC in cervical cancer patients. Stably transfected FGFR2 IIIc in CaSki cells induced the growth of cells in vitro and in vivo.

\section{Materials and methods}

Materials. The following were purchased: recombinant human FGFR $2 \alpha(\mathrm{IIIb}) / \mathrm{Fc}$ and FGFR $2 \alpha($ IIIc)/Fc chimera proteins from R\&D Systems, Inc. (Minneapolis, MN, USA); horseradish peroxidase (HRP)-conjugated goat anti-rabbit IgG secondary antibodies from American Qualex (San Clemente, CA, USA); rabbit polyclonal anti-human IgG and mouse monoclonal anti-Ki-67 antibodies from Dako Japan (Tokyo, Japan); Immobilon P transfer membrane from Millipore (Yonezawa, Japan); Super Signal West Dura chemiluminescent substrates from Thermo Fisher Scientific, Inc. (Waltham, MA, USA); Alexa 488-conjugated goat antirabbit IgG and pcDNA 3.1(-) or (+) vector from Invitrogen (Carlsbad, CA, USA); RNAiso Plus and a FastPure RNA kit from Takara Bio, Inc. (Shiga, Japan); a digoxigenin (DIG) nucleic acid detection kit and a DIG RNA labeling kit from Roche Diagnostics GmbH (Mannheim, Germany); a High Capacity cDNA Reverse Transcription kit, TaqMan Universal PCR Master Mix and a TaqMan MGB probe for ribosomal eukaryotic 18S rRNA from Applied Biosystems (Foster City, CA, USA); Histofine Simple Stain MAX PO (R) or (M) kits from Nichirei (Tokyo, Japan); Matsunami Adhesive Slide (MAS) and 4-well glass bottom dishes from Matsunami Glass Ind., Ltd. (Osaka, Japan); Vectashield mounting medium containing 4',6-diamidino-2-phenylindole dihydrochloride (DAPI) from Vector Laboratories, Inc. (Burlingame, CA, USA); pIRES2-EGFP vector from Clontech (Palo Alto, CA, USA); geneticin from Gibco BRL (Grand Island, NY, USA); WST-8 cell counting kit from Wako Pure Chemical Industries (Osaka, Japan). All other chemicals and reagents
Table I. Clinical features of cervical cancer patients.

\begin{tabular}{lr}
\hline Age (years) & $29-72$ \\
Median & 55 \\
Mean & 53 \\
FIGO stage & \\
Ia & 5 \\
Ib & 13 \\
IIa & 1 \\
IIb & 10
\end{tabular}

$\begin{array}{cr}\text { Keratinization } & \\ \text { Absent } & 21 \\ \text { Present } & 8\end{array}$

Nodal metastasis

Absent 20

Present 9

HPV infection

Absent 1

Present 19

Not examined 9

were purchased from Sigma Chemical Corp. (St. Louis, MO, USA).

Non-cancerous, CIN and cancerous cervical tissues. Cervical cancer samples (29 patients; mean age, 53 years; median age, 55; range, 29-72 years) were obtained from patients undergoing surgery for invasive cervical cancer at the Department of Obstetrics and Gynecology of Nippon Medical School Hospital (Bunkyo-ku, Tokyo, Japan) from 2005 to 2008 (Table I). On the basis of the classification of the International Federation of Gynecology and Obstetrics (FIGO), the stages of the patients were classified as follows: stage Ia, 5; stage Ib, 13; stage IIa, 1; stage IIb, 10. On the basis of the World Health Organization (WHO) Classification of Tumors, the patients were classified into the keratinizing type (8 patients) and non-keratinizing type (21 patients) of SCC. Fifteen samples each for CINs 1-3 were obtained by cervical biopsies at the same hospital and used for this study. For control samples, cervical biopsies from patients with chronic cervicitis (10 patients) were used. Paraffin-embedded specimens were prepared for immunohistochemical analysis as previously described (31). This study was carried out following the ethics guidelines of Nippon Medical School and the principles embodied in the Declaration of Helsinki, 1975.

Human cervical cancer cell lines. ME-180 cells were obtained from the Cell Resource Center for Biomedical Research, Institute of Development, Aging and Cancer, Tohoku University (Sendai, Japan). CaSki cells were obtained from RIKEN BioResource Center (Ibaraki, Japan). CaSki and ME-180 cells were grown in RPMI-1640 medium containing $10 \%$ heat-inactivated fetal bovine serum (FBS), $200 \mathrm{U} / \mathrm{ml}$ 
penicillin and $200 \mu \mathrm{g} / \mathrm{ml}$ kanamycin at $37^{\circ} \mathrm{C}$ under a humidified $5 \% \mathrm{CO}_{2}$ atmosphere.

Rabbit polyclonal anti-human FGFR2 IIIc antibody. To confirm the expression of FGFR2 IIIc at the protein level in cervical cancer, a specific antibody against human FGFR2 IIIc was prepared. The anti-FGFR2 IIIc antibody used was an affinity-purified rabbit polyclonal antibody raised against a peptide corresponding to the amino acids of the human FGFR2 IIIc protein (accession no. NM_000141). The peptide sequence 'AAGVNTTDKEIEVLYIR' is located at the carboxyl-terminal half of the Ig loop closest to the transmembrane region that is specific for FGFR2 IIIc. To estimate the reactivity of the anti-FGFR2 IIIc antibody, FGFR $2 \alpha(\mathrm{IIIb}) / \mathrm{Fc}$ and FGFR $2 \alpha($ IIIc)/Fc chimera proteins were subjected to sodium dodecyl sulfate-polyacrylamide gel electrophoresis (SDS-PAGE), and the separated proteins were transferred to Immobilon $P$ transfer membranes, which were then incubated overnight with the anti-FGFR2 IIIc antibody (1:6000 dilution) at $4^{\circ} \mathrm{C}$. The membranes were washed and incubated with HRP-conjugated anti-rabbit IgG antibody for $60 \mathrm{~min}$ at room temperature (RT). After washing, the blot was visualized by enhanced chemiluminescence. The membrane was reblotted with rabbit polyclonal anti-human IgG antibody to confirm the equal loading.

Immunohistochemistry. Paraffin-embedded sections (3 $\mu \mathrm{m})$ were subjected to immunostaining using a Histofine Simple Stain MAX PO (R) kit for FGFR2 IIIc and MAX PO(M) kit for Ki-67. After deparaffinization, the tissue sections for $\mathrm{Ki}-67$ immunostaining were preheated in $10 \mathrm{mM}$ citrate buffer (pH 6.0) for $15 \mathrm{~min}$ at $121^{\circ} \mathrm{C}$ in an autoclave oven. Then, endogenous peroxidase activity was blocked by incubation for $30 \mathrm{~min}$ with $0.3 \%$ hydrogen peroxide in methanol. The tissue sections were then incubated with the anti-FGFR2 IIIc antibody (1:200 dilution) or anti-Ki-67 antibody (1:100 dilution) in phosphate-buffered saline (PBS) containing $1 \%$ bovine serum albumin (BSA) for $16 \mathrm{~h}$ at RT. Bound antibodies were detected with the Simple Stain MAX PO (R) or (M) reagent, using diaminobenzidine tetrahydrochloride as the substrate. The sections were then counterstained with Mayer's hematoxylin. Negative control tissue sections were prepared by omitting the primary antibody.

Probe preparation. A Bam $\mathrm{HI}-E c o \mathrm{RI}-a d a p t o r-c o n j u g a t e d$ cDNA fragment, corresponding to nucleotides 1591-1740 of the human FGFR2 IIIc cDNA sequence (150 bp, accession no. NM_000141), was generated by PCR amplification of single-stranded cDNA that was reverse-transcribed from human fibroblast RNA as previously reported (32). The human FGFR2 IIIc cDNA fragments were subcloned into the pcDNA 3.1(-) or (+) vector and the authenticity was confirmed by sequencing. The antisense or sense probes were labeled with digoxigenin-UTP using T7 RNA polymerase from the DIG RNA-labeling kit.

In situ hybridization (ISH) analysis. ISH was performed as previously reported (33-35). Tissue sections were deparaffinized and incubated at RT for 20 min with $0.2 \mathrm{~N} \mathrm{HCl}$ and then at $37^{\circ} \mathrm{C}$ for $15 \mathrm{~min}$ with $100 \mu \mathrm{g} / \mathrm{ml}$ proteinase $\mathrm{K}$. The sections were then postfixed for 5 min in PBS containing $4 \%$ paraformaldehyde (PFA), and incubated twice for $15 \mathrm{~min}$ each with PBS containing $2 \mathrm{mg} / \mathrm{ml}$ glycine at RT and once in $50 \%(\mathrm{v} / \mathrm{v})$ formamide/2X SSC for $1 \mathrm{~h}$ at $42^{\circ} \mathrm{C}$ prior to the initiation of the hybridization reaction. The hybridization buffer contained $0.6 \mathrm{M} \mathrm{NaCl}, 1 \mathrm{mM}$ EDTA, $10 \mathrm{mM}$ Tris- $\mathrm{HCl}$ (pH 7.6), 0.25\% SDS, 200 mg/ml yeast tRNA, IX Denhard's solution, $10 \%$ dextran sulfate, $40 \%$ formamide and $200 \mathrm{ng} / \mathrm{ml}$ of the indicated digoxigenin-labeled riboprobe. Hybridization was performed in a moist chamber for $16 \mathrm{~h}$ at $42^{\circ} \mathrm{C}$. The sections were then washed sequentially with $2 \mathrm{X} \mathrm{SSC} \mathrm{for}$ $20 \mathrm{~min}$ at $42^{\circ} \mathrm{C}$, followed by $0.2 \mathrm{X} \mathrm{SSC}$ for $20 \mathrm{~min}$ at $42^{\circ} \mathrm{C}$. For immunological detection, the DIG nucleic acid detection kit was used. The sections were washed briefly with buffer 1 (100 mM Tris- $\mathrm{HCl}$ and $150 \mathrm{mM} \mathrm{NaCl}, \mathrm{pH} \mathrm{7.5),} \mathrm{incubated}$ with $1 \%(\mathrm{w} / \mathrm{v})$ blocking reagents in buffer 1 for $60 \mathrm{~min}$ at RT, and with alkaline-phosphatase-conjugated polyclonal sheep anti-digoxigenin Fab fragment containing 0.2\% Tween-20 at 1:2000 dilution for $60 \mathrm{~min}$ at RT. The sections were then washed twice for $15 \mathrm{~min}$ at RT with buffer 1 containing $0.2 \%$ Tween-20, equilibrated with buffer $3(100 \mathrm{mM}$ Tris- $\mathrm{HCl}, 100 \mathrm{mM} \mathrm{NaCl}, 50 \mathrm{mM} \mathrm{MgCl}$, $\mathrm{pH}$ 9.5) for $2 \mathrm{~min}$, and incubated with a staining solution containing nitroblue tetrazolium and X-phosphate in a dark box for $1 \mathrm{~h}$. After the reaction was stopped with $\mathrm{TE}$ buffer $(10 \mathrm{mM}$ Tris- $\mathrm{HCl}$ and $1 \mathrm{mM}$ EDTA, pH 8.0), the sections were mounted in an aqueous mounting medium.

Quantitative real-time PCR of FGFR2 IIIc in cervical cancer cell lines. The cervical cancer cells were grown in RPMI-1640 medium with 10\% FBS for $48 \mathrm{~h}$. Total RNA extraction from cervical cancer cells was performed using RNAiso Plus and then purified with a FastPure RNA kit. Then, cDNA synthesis was performed using High Capacity cDNA Reverse Transcription kit following the manufacturer's protocol. Quantitative real-time PCR (Q-PCR) was performed using the ABI PRISM 7900HT Sequence Detection System. The real-time PCR primers used for FGFR2 IIIc are nucleotides (nts) 1693-1716 (5'-GGA-TAT-CCT-TTC-ACTCTG-CAT-GGT-3') and nts 1770-1794 (5'-TGC-AGT-AAATGG-CTA-TCT-CCA-GGT-A-3') of the human FGFR2 IIIc cDNA (102 bp, accession no. NM_000141.) As TaqMan probes, 5'-CAG-TTC-TGC-CAG-CGC-CTG-GAA-GA-3' was used for FGFR2 IIIc. The PCR reaction mixture $(50 \mu \mathrm{l})$ contained $2 \mu \mathrm{l}$ of template cDNA, $0.9 \mu \mathrm{M}$ primers, $0.25 \mu \mathrm{M}$ probe and $25 \mu 1$ of TaqMan Universal PCR Master Mix. $18 \mathrm{~S}$ rRNA, as the internal positive control, was amplified using the TaqMan MGB probe for ribosomal eukaryotic $18 \mathrm{~S}$ rRNA. The optimized program included uracil Nglycosylase incubation at $50^{\circ} \mathrm{C}$ for $2 \mathrm{~min}$ and AmpliTaq Gold activation at $95^{\circ} \mathrm{C}$ for $10 \mathrm{~min}$, followed by 50 cycles of amplification at $95^{\circ} \mathrm{C}$ for $15 \mathrm{sec}$ and at $60^{\circ} \mathrm{C}$ for $60 \mathrm{sec}$ for FGFR2 IIIc and RS-18. Results were expressed as target/RS-18 as an internal standard concentration ratio. Gene expression measurements were performed in triplicate.

Immunofluorescence staining and confocal laser microscopy. The same anti-FGFR2 IIIc antibody used for the immunohistochemistry was also used for immunofluorescence staining of ME-180 and CaSki cells. The cells were incubated with 
the anti-FGFR2 IIIc antibody (1:50) in PBS containing $1 \%$ BSA for $18 \mathrm{~h}$ at $4^{\circ} \mathrm{C}$. For negative control, the cells were incubated with PBS containing $1 \%$ BSA. The cells were washed with PBS and then incubated with Alexa 488conjugated goat anti-rabbit IgG. One hour after incubation, the cells were washed with PBS, and then mounted with Vectashield mounting medium containing DAPI. Fluorescent images were acquired using a confocal laser scanning microscope Digital Eclipse TE 2000-E (Nikon Insteck Co., Ltd., Tokyo, Japan) and a 100X immersion lens (Nikon Palm Apo VC) with blue diode (BD) and helium-neon lasers and were analyzed using the confocal microscope Digital Eclipse C1 control software EZ-C1 (version 2.30) (Nikon Insteck). The excitation wavelength for Alexa 488 was $488 \mathrm{~nm}$, and the emission was selected and recorded using a 500- to 530-nm band-pass filter. In addition, the excitation wavelength for DAPI was $405 \mathrm{~nm}$, and the emission was selected and recorded using a 432- to 446-nm band-pass filter.

Construction of FGFR2 IIIc expression vector and generation of stably transfected clones. Full-length FGFR2 IIIc cDNA fragment was ligated to the 3 '-end of the human cytomegalovirus early promoter/enhancer in pIRES2-EGFP, a eukaryotic expression vector. Proper orientation of the insert was verified by DNA sequencing. CaSki cells $\left(\sim 1 \times 10^{5} / \mathrm{ml}\right)$ were transfected with $3 \mu \mathrm{g}$ of DNA using FuGene HD, and the cells were passaged and cultured with geneticin $(500 \mu \mathrm{g} /$ $\mathrm{ml}$ ). Independent colonies were isolated by ring cloning, transferred to microtiter wells, and expanded in geneticin $(250 \mu \mathrm{g} / \mathrm{ml})$.

Morphological analysis of FGFR2 IIIc-transfected CaSki cells. Stable FGFR2 IIIc-transfected CaSki cells, emptyvector-transfected cells (Mock cells) and non-treated cells (wild-type) were incubated in RPMI-1640 medium with $10 \%$ FBS for $48 \mathrm{~h}$. The morphology of the cells was observed using a phase-contrast microscope (Nikon Eclipse TE2000-U).

Anchorage-dependent growth assays. To examine the growth rates of FGFR2 IIIc-transfected clones, a non-radioactive cell proliferation assay was performed. The cells were cultured in RPMI-1640 medium with $10 \%$ FBS at a density of $1 \times 10^{3}$ in 96-well plates followed by incubation at $37^{\circ} \mathrm{C}$ in a humidified 5\% $\mathrm{CO}_{2}$ atmosphere for 24, 48, 72 and $96 \mathrm{~h}$. Then, the cells were incubated with WST- 8 cell counting reagent for $4 \mathrm{~h}$ at $37^{\circ} \mathrm{C}$ and the optical density of the culture solution in the plate was measured using an ELISA plate reader (Bio-Rad Laboratory, Hercules, CA) at $450 \mathrm{~nm}$. The experiments were performed in triplicate.

Single-cell movement assay. CaSki cells (5000 per well) were seeded onto a 4-well glass bottom dish and grown for $48 \mathrm{~h}$. Cell movement was then monitored for $24 \mathrm{~h}$ using a Digital Eclipse TE 2000-E motorized inverted microscope taking pictures every $5 \mathrm{~min}$. The total distance of individual cells covered within $24 \mathrm{~h}$ was determined using the Metamorph software 7.6 (Universal Imaging Corp., Ltd., Buckinghamshire, UK).

In vivo tumorigenicity assay. To assess the effect of FGFR2 IIIc expression on tumorigenicity, $3 \times 10^{6}$ cells/animal were

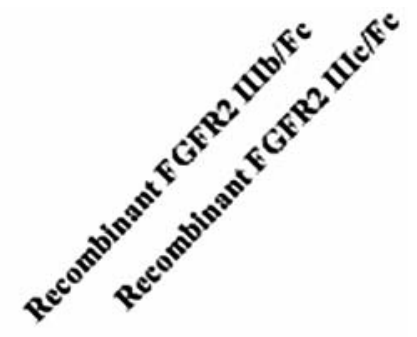

\section{Anti-FGFR2 IIIc antibody}

\section{Anti-IgG antibody}

Figure 1. Preparation of rabbit polyclonal anti-FGFR2 IIIc antibody. Polyclonal anti-FGFR2 IIIc antibody reacted with recombinant FGFR $2 \alpha($ IIIc)/ Fc chimera proteins (upper panel, right lane), while the antibody did not react with recombinant FGFR $2 \alpha(\mathrm{IIIb}) / \mathrm{Fc}$ chimera proteins (upper panel, left lane). Loading control of anti-human IgG antibody reacted with each recombinant protein on the reblotted membrane (lower panel).

injected into 6-week-old, female, athymic (nude) mice $(n=17)$. The CaSki cells were injected subcutaneously on both sides of the back. The animals were monitored for tumor formation thrice weekly and sacrificed 30 days after injection. Tumor volume was calculated using the formula: volume $=a \times b^{2} \times 0.5$, where $a$ is the longest diameter and $b$ the shortest.

Statistical analysis. All quantitative data are presented as mean \pm SE and assessed using Student's t-test and MannWhitney test. Computations were performed using the Stat View J version 5.0 software package (SAS Institute, Inc., Cary, NC).

\section{Results}

Western blot analysis of anti-FGFR2 IIIc antibody. To examine the FGFR2 IIIc expression at the protein level in cervical cancer, we prepared rabbit polyclonal anti-human FGFR2 IIIc antibody. FGFR2 IIIc and FGFR2 IIIb have high amino acid homology; thus, we examined the specificity of the antibody to FGFR2 IIIc using recombinant FGFR2 IIIc and IIIb proteins. Anti-FGFR2 IIIc antibody reacted with recombinant FGFR $2 \alpha$ (IIIc)/Fc chimera proteins, which migrated at approximately 100-110 kD (Fig. 1, upper panel, right lane). In contrast, the antibody did not cross-react with recombinant FGFR $2 \alpha(\mathrm{III}) / \mathrm{Fc}$ chimera proteins (Fig. 1, upper panel, left lane). Anti-human $\mathrm{IgG}$ antibody reacted with the Fc regions of each recombinant protein on the reblotted membrane (Fig. 1, lower panel). These findings indicate that the anti-FGFR2 IIIc antibody is highly specific to FGFR2 IIIc.

Immunohistochemical and ISH analyses of FGFR2 IIIC in non-cancerous cervical tissues. Immunohistochemical and 


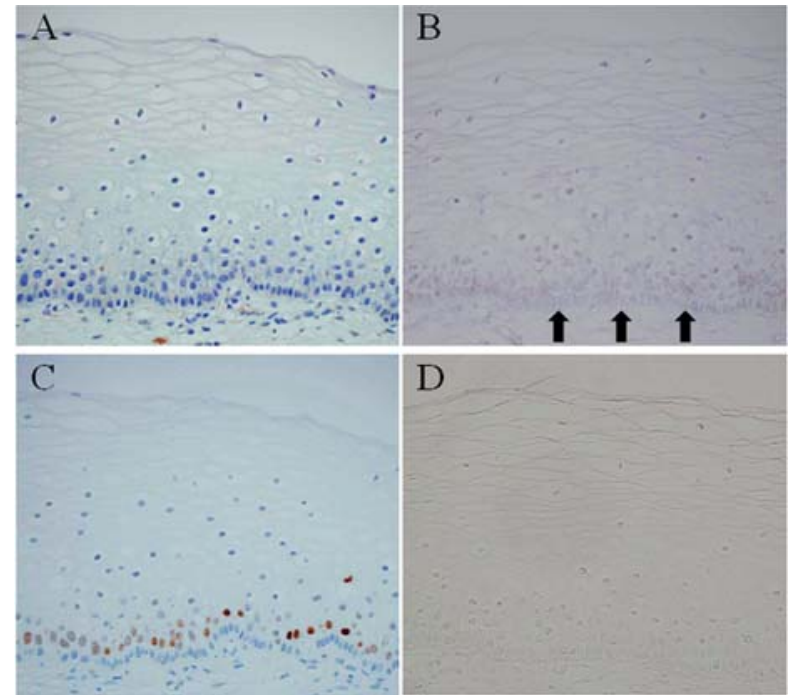

Figure 2. Immunohistochemical and ISH analyses of FGFR2 IIIc in noncancerous cervical tissues. Immunohistochemically, the FGFR2 IIIc protein was not or very faintly localized in squamous epithelial cells (A) and ISH showed that the FGFR2 IIIc mRNA was expressed in basal cells of squamous epithelium [(B), arrows]. The cell proliferation marker, Ki-67, was localized in parabasal cells in non-cancerous squamous epithelium (C). The sense probe did not detect any signals (D). Immunohistochemistry, (A) and (C); in situ hybridization, (B) and (D); original magnification, (A)-(D) x400 in situ hybridization analyses were performed to examine the localization of FGFR2 IIIc protein and its mRNA in cervical tissues. In non-cancerous cervical tissues, the FGFR2 IIIc protein was not or very faintly localized in squamous epithelial cells (Fig. 2A) and its mRNA was expressed in basal cells of squamous epithelium (Fig. 2B, arrows). Ki-67 was localized in parabasal cells in non-cancerous squamous epithelium (Fig. 2C). The sense probe did not detect any signals (Fig. 2D).

Immunohistochemical and ISH analyses of FGFR2 IIIc in CIN tissues. In CIN 1, FGFR2 IIIc and its mRNA were detected in the basal to one-third of the epithelial layers (Fig. 3A and D, respectively). Ki-67 was localized at the parabasal to lower one-third of the epithelial layers (Fig. 3G). In CIN 2, FGFR2 IIIc, its mRNA and Ki-67 were localized between the basal layer and two-thirds of the squamous epithelial layers (Fig. 3B, E and H, respectively). In CIN 3, FGFR2 IIIc, its mRNA and Ki-67 were strongly expressed in most squamous epithelial layers, except for the superficial layers (Fig. 3C, F and I, respectively). The sense probes did not yield any positive signals (Fig. 3J-L).

Immunohistochemical and ISH analyses of FGFR2 IIIc in cervical cancer tissues. In cervical cancer tissues, the non-

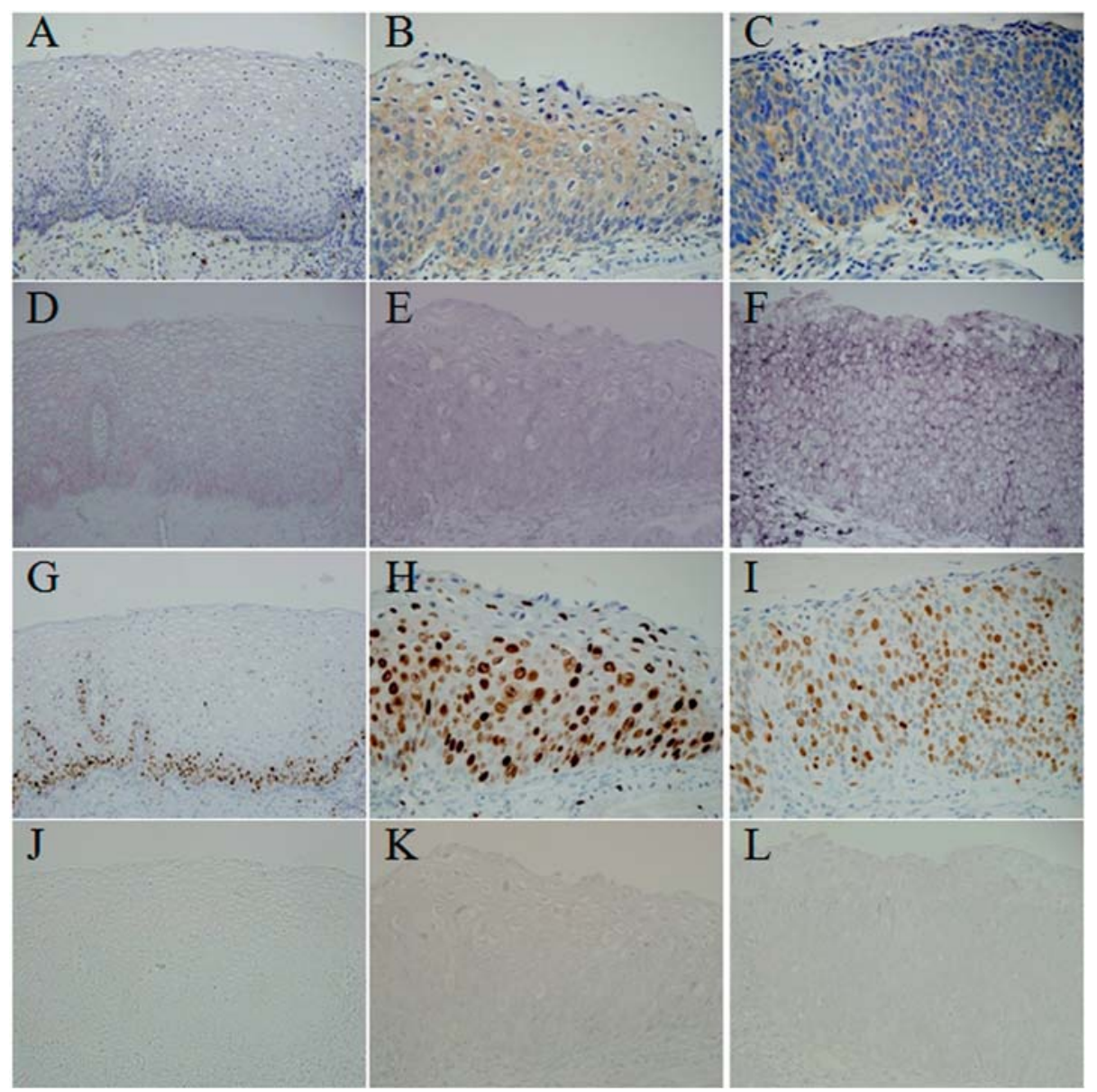

Figure 3. Immunohistochemical and ISH analyses of FGFR2 IIIc in CIN. In CIN 1, FGFR2 IIIc and its mRNA were localized at the basal to lower one-third of squamous epithelial layers [(A) and (D)], and Ki-67 was detected in the parabasal to one-third of the epithelial layers (G). In CIN 2, FGFR2 IIIc, its mRNA and Ki-67 were localized between the basal layer and two-thirds of the squamous epithelial layers [(B), (E) and (H), respectively]. In CIN 3, FGFR2 IIIc, its mRNA and Ki-67 were strongly expressed in most squamous epithelial layers, except for the superficial layers [(C), (F) and (I), respectively]. The sense probes did not show positive signals (J)-(L). Immunohistochemistry, (A)-(C) and (G)-(I); in situ hybridization, (D)-(F) and (J)-(L); original magnification, (A), (D), (G) and (J) x200; (B), (C), (E), (F), (H), (I), (K) and (L) x400. 


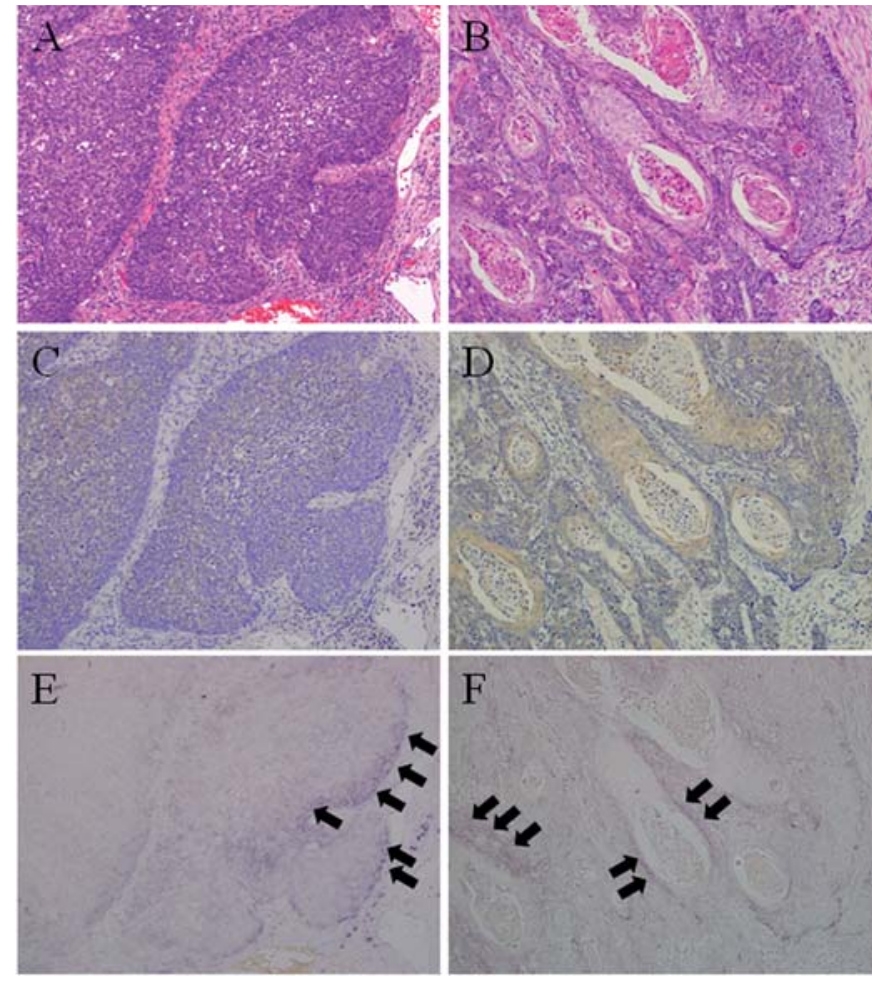

Figure 4. Immunohistochemical and ISH analyses of FGFR2 IIIc in cervical cancer The non-keratinizing type of cancer showed solid cancer cell proliferation and the keratinizing type of cancer showed keratinizing foci in cancer cell nests [(A) and (B), respectively]. Immunohistochemically, the FGFR2 IIIc protein was diffusely localized in cancer cell nests in the nonkeratinizing type of cells, and strongly expressed in the central portions with keratinization in the keratinizing type [(C) and (D), respectively]. Moreover, the FGFR2 IIIc mRNA was strongly expressed in the invasive front of cancer cell nests $[(\mathrm{E})$ and $(\mathrm{F})$, arrows]. H\&E, (A) and (B); immunohistochemistry, (C) and (D); in situ hybridization, (E) and (F); original magnification, (A)-(F) $\times 200$.

keratinizing type of cancer showed alveolar patterns with solid cancer cell proliferation and the keratinizing type of cancer showed keratinizing foci in cancer cell nests (Fig. 4A and $\mathrm{B}$, respectively). Immunohistochemically, the FGFR2 IIIc protein was detected in cancer cells of the non-keratinizing and keratinizing types in all the examined cervical cancer patients ( 21 and 8 patients, Fig. $4 \mathrm{C}$ and D, respectively). The FGFR2 IIIc protein was diffusely localized in cancer cell nests in the non-keratinizing type of cells, and strongly expressed in the central portions with keratinization in the keratinizing type. Moreover, FGFR2 IIIc mRNA was strongly expressed in cancer cells in the peripheral lesion of cancer cell nests in both types of cancer (Fig. 4E and F arrows, respectively).

Expression of FGFR2 IIIc in cervical cancer cell lines. To examine whether cervical cancer cells express FGFR2 IIIc, Q-PCR was performed. FGFR2 IIIc mRNA was expressed in two cervical cancer cell lines. The expression level of FGFR2 IIIc mRNA was higher in CaSki cells than in ME-180 cells (Fig. 5). Immunofluorescent analysis showed that the FGFR2 IIIc protein is expressed in the cell membrane and cytoplasm of ME-180 and CaSki cells (Fig. 6A and B) and the FGFR2 IIIc level was higher in CaSki cells than in ME-180 cells.

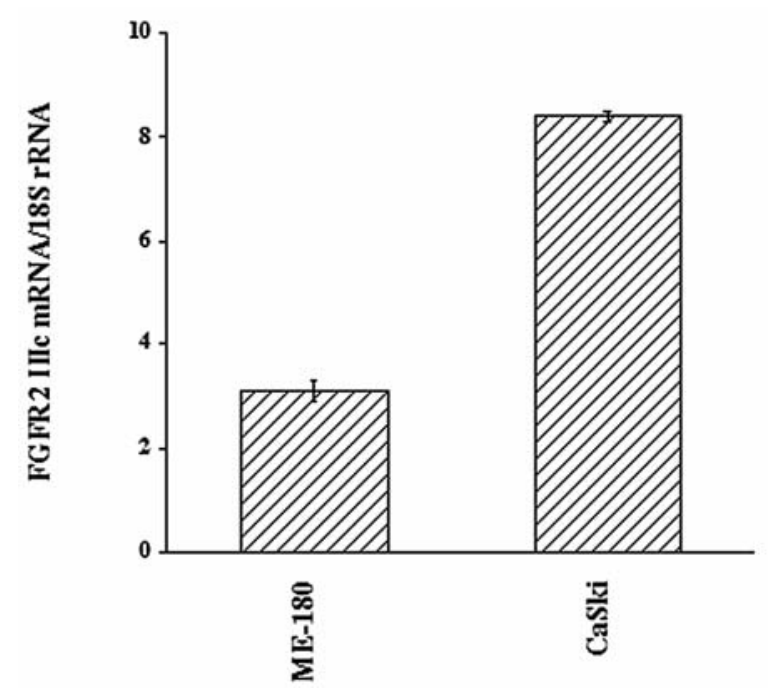

Figure 5. Expression of FGFR2 IIIc mRNA in cervical cancer cell lines. FGFR2 IIIc mRNA was expressed in CaSki and ME-180 cervical cancer cell lines. The expression level of FGFR2 IIIc mRNA was higher in CaSki cells than in ME-180 cells.
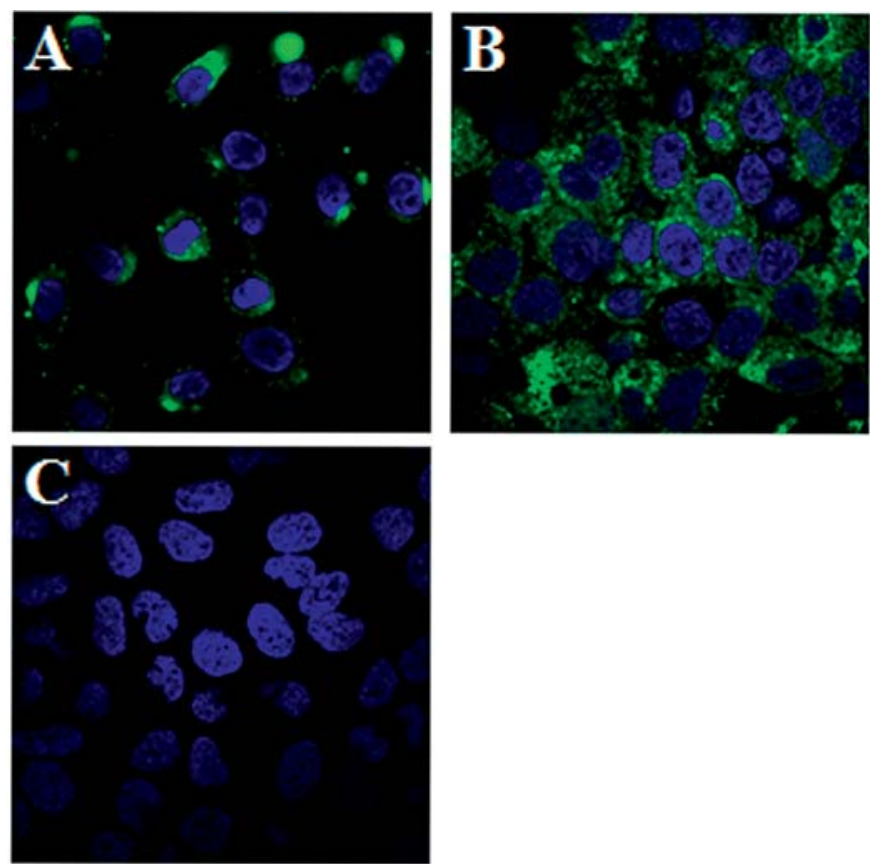

Figure 6. Immunofluorescence analysis of FGFR2 IIIc in cervical cancer cell lines. Immunofluorescence analysis showed that the FGFR2 IIIc protein is expressed in ME-180 (A) and CaSki cells (B) and the FGFR2 IIIc was more prominently localized in CaSki cells than in ME-180 cells. Negative control without primary antibody showed no positive staining (C). Original magnification, (A)-(C) x1000.

Negative control without primary antibody showed no positive staining (Fig. 6C).

Stable FGFR2 IIIc-transfected CaSki cells. To examine the role of FGFR2 IIIc in CaSki cells, we prepared FGFR2 IIIcoverexpressing cervical cancer cells. A full-length FGFR2 IIIc cDNA was subcloned into pIRES2-EGFP vector and stably transfected in these cells. The expression levels of 


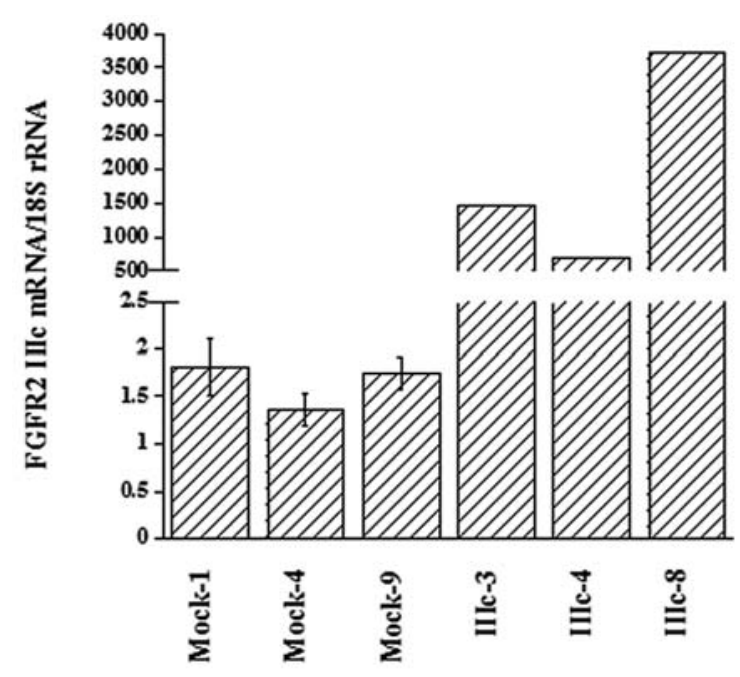

Figure 7. Quantitative real-time PCR analysis of FGFR2 IIIc mRNA in FGFR2 IIIc-transfected CaSki cells. The expression levels of FGFR2 IIIc mRNA in Mock and FGFR2 IIIc-transfected CaSki cells were examined by Q-PCR. FGFR2 IIIc mRNA/18S rRNA levels were high in three clones (IIIc-3, IIIc-4 and IIIc-8), whereas they were low in Mock cells (Mocks-1, -4 and -9$)$.
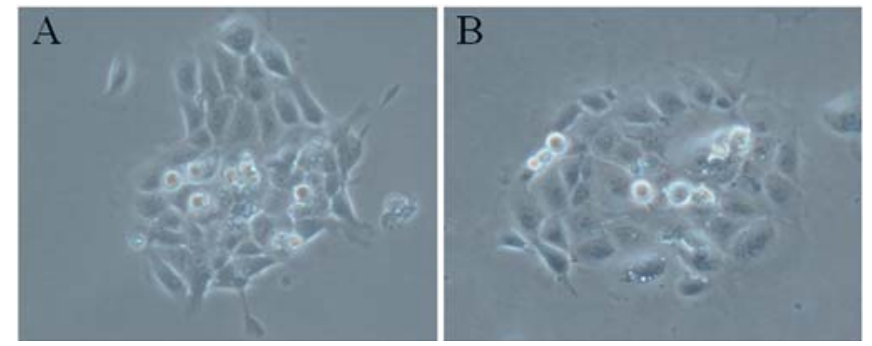

Figure 8. Morphological features of FGFR2 IIIc-transfected CaSki cells FGFR2 IIIc-transfected CaSki cells showed no characteristic histological alterations as compared with Mock cells, (A) and (B). Phase-contrast microscopy; original magnification, x200.

FGFR2 IIIc mRNA in Mock and FGFR2 IIIc-transfected CaSki cells were examined by Q-PCR. FGFR2 IIIc mRNA/ $18 \mathrm{~S}$ rRNA levels were high in three clones (Fig. 7; IIIc-3, IIIc-4 and IIIc-8), whereas they were low in Mock cells (Mocks-1, 4 and 9). The expression level of FGFR2 IIIc mRNA was $>500$-fold higher in FGFR2 IIIc-transfected CaSki cells than in Mock cells.

Morphological alteration of FGFR2 IIIc-transfected CaSki cells. The FGFR2 IIIc-transfected CaSki cells did not show characteristic morphological alterations as compared with Mock cells (Fig. 8). The cells possessed polygonal or short spindle-like cytoplasm with branched projections.

Cell proliferation assay of FGFR2 IIIc-transfected CaSki cells in vitro. The FGFR2 IIIc-transfected CaSki and Mock cells were cultured in RPMI-1640 medium containing 10\% FBS for 24, 48, 72, 96 and $120 \mathrm{~h}$. Three clones of stable FGFR2 IIIc-transfected CaSki cells (Fig. 9; IIIc-3, IIIc-4 and IIIc-8) showed an increase in proliferation as compared with Mock cells (Mocks-1, 4 and 9). After 96 h, all three clones of FGFR2 IIIc-transfected cells showed significantly higher growth rates than the three Mock clones $(\mathrm{P}<0.002)$.

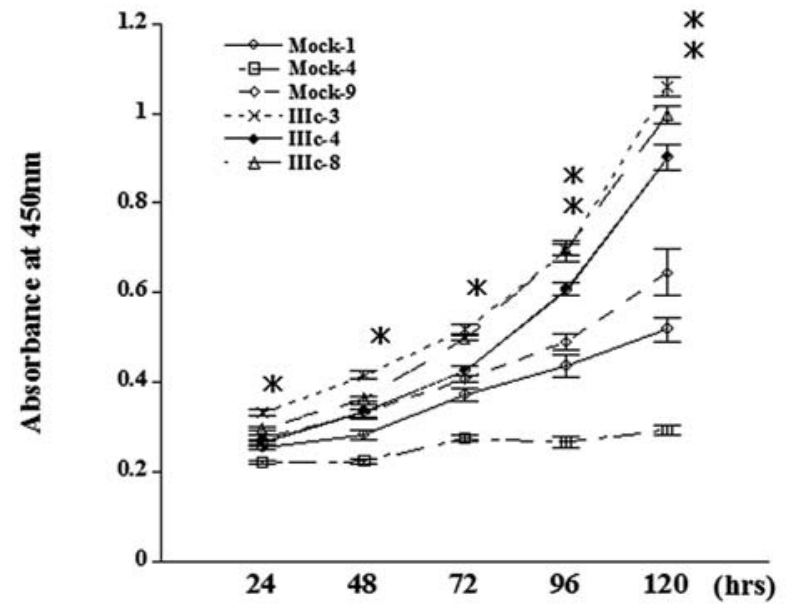

Figure 9. Cell proliferation assay of FGFR2 IIIc-transfected CaSki cells The CaSki cells were cultured in RPMI-1640 medium containing 10\% FBS for 24, 48, 72, 96 and $120 \mathrm{~h}$. The growth rates of stable FGFR2 IIIctransfected CaSki cells (IIIc-3, IIIc-4 and IIIc-8) were higher than those of the Mock cells (Mocks-1, 4 and 9). "IIIc-3 and IIIc-8 vs. Mocks-1, -4 and -9, $\mathrm{P}<0.02 ;{ }^{* *}$ IIIc-3, IIIc-4 and IIIc- 8 vs. Mocks-1, -4 and $-9, \mathrm{P}<0.002$.
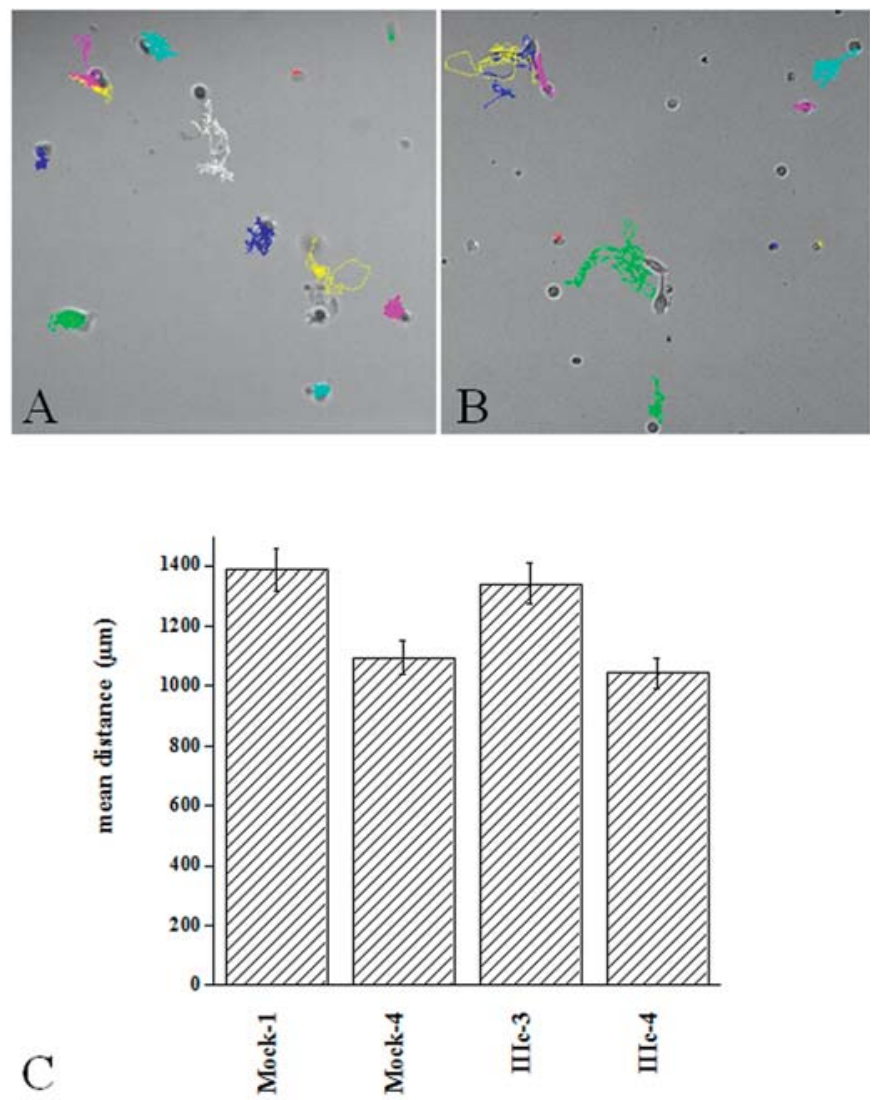

Figure 10. Single-cell movement assay. Single-cell movements of Mock and FGFR2 IIIc-transfected cells were monitored by time-lapse microscopy [(A) and (B), respectively]. The distances covered within $24 \mathrm{~h}$ by Mocks- 1 and -4 and IIIc-3 and IIIc-4 cells showed no statistical difference (C).

Single-cell movement assay. Single-cell movement was characterized next to determine whether FGFR2 IIIc is involved in cellular migration. Single-cell movement was monitored by time-lapse microscopy (Fig. 10A and B). The distances covered within $24 \mathrm{~h}$ were 1388 and $1096 \mu \mathrm{m}$ for Mocks-1 and 4, respectively. Expression of FGFR2 IIIc resulted 


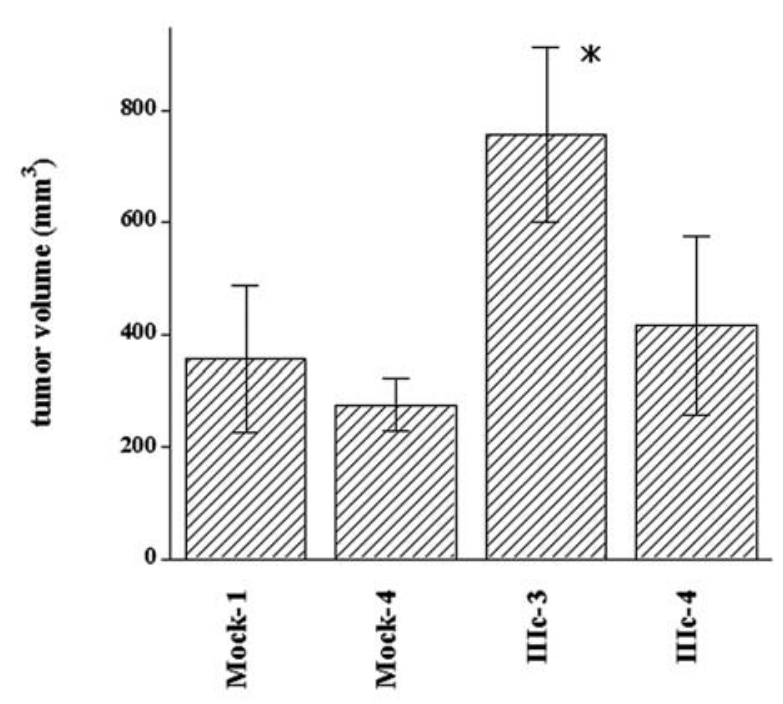

Figure 11. FGFR2 IIIc-transfected CaSki cells in nude mice. FGFR2 IIIctransfected CaSki cells formed larger subcutaneous tumors than Mock cells 30 days after the subcutaneous injection of the cancer cells in nude mice. IIIc- 3 cells showed a more significantly increased growth than Mock- 4 cells. *IIIc-3 vs. Mock-4, $\mathrm{P}<0.007$.

in the same motility in IIIc-3 and IIIc-4 cells with covered distances of 1342 and $1044 \mu \mathrm{m}$, respectively (Fig. 10C). There was no significant difference between FGFR2 IIIc-transfected and Mock cells. We also performed Boyden chamber assay to assess the cell movement, but there was no significant difference between FGFR2 IIIc-transfected and Mock cells (data not shown).

In vivo growth characteristics of FGFR2 IIIc-transfected CaSki cells. Next, we examined whether FGFR2 IIIc expression in cervical cancer cells is associated with increased tumor growth in nude mice. FGFR2 IIIc-transfected CaSki cells formed larger subcutaneous tumors than Mock cells 30 days after the subcutaneous injection of the cancer cells in nude mice (Fig. 11). IIIc-3 cells showed a more significantly increased growth than Mock-4 cells $(\mathrm{P}<0.007)$.

\section{Discussion}

In cancer cells, the increased expression level of FGFR2 IIIc is considered to correlate with cancer aggressiveness $(24,25)$. To examine the localization of FGFR2 IIIc in cervical tissues and cancer cell lines, we prepared highly specific anti-FGFR2 IIIc antibodies. This antibody was raised against a peptide mapping at the IIIc region of FGFR2 IIIc. The amino acid sequences of the IIIc region of FGFR2 IIIc and IIIb are highly homologous, but this antibody did not cross-react with FGFR2 IIIb or FGFR1 IIIb (data not shown).

In the case of non-cancerous cervical tissues, we previously reported that FGFR2 IIIb is weakly expressed on the surface of squamous epithelial cells (5). Our present study indicates that FGFR2 IIIc is not or very weakly localized in the basal layer of the epithelium in non-cancerous cervical tissues. The different expression patterns of FGFR2 IIIb and FGFR2 IIIc isoforms in normal cervical epithelium may indicate that FGFR2 IIIb plays a role in cell differentiation or apoptosis, whereas FGFR2 IIIc affects epithelial cell growth.
A large number of growth factors and their receptors were reported to be expressed in CIN tissues. A significant positive correlation was found between VEGF-A, VEGF-C, VEGF-D and VEGFR-3 expressions with the different stages of cervical carcinogenesis (36). Significant differences in protein expressions for VEGF-C, VEGF-D and VEGFR-3 were found between CINs 1-2 and CIN 3, but not between CIN 3 and cervical cancer (6). CINs 2-3 exhibited higher VEGF mRNA, transforming growth factor (TGF)- 11 and TGF- $\beta$ receptor 1 levels than CIN 1 (37). The expression levels of insulin-like growth factor 1 receptor (IGF-1R) and phosphorylated IGFR-1 were positively correlated with the CIN grades (7). The expressions and roles of FGF and FGFR members have not been reported. In this study, we found that FGFR2 IIIc is clearly expressed in dysplastic or neoplastic cells in CIN. FGFR2 IIIc expression patterns in CIN were similar to the expression pattern of Ki-67, one of the reliable cell proliferation markers. These findings may indicate that FGFR2 IIIc expression is closely related to the growth of neoplastic cells in CIN and carcinogenesis.

In cervical cancer tissues, FGFR2 IIIb was detected in $94 \%$ of patients with keratinizing type and $79 \%$ of patients with non-keratinizing type of cervical cancer (5). Furthermore, FGFR2 IIIb expression was detected in CaSki cells but not in ME-180 cells. CaSki cells have a large and pleomorphic cytoplasm and their histological appearance is similar to that of well-differentiated SCC, whereas ME-180 cells have a round or oval cytoplasm and a high nuclear/cytoplasm ratio similar to that of poorly differentiated SCC. In contrast, FGFR2 IIIc was expressed in all cervical cancer patients including keratinizing and non-keratinizing types and it was expressed in both CaSki and ME-180 cells. These results suggest that most patients with cervical cancer show overexpression of both FGFR2 IIIb and IIIc isoforms. The reason for the FGFR2 IIIc overexpression in cervical cancer is not yet clear, but gene amplification, mutation or spliceosome dysregulation of FGFR2 might occur in the cancer cells. HPV infection was detected in $99 \%$ of invasive SCC of the cervix, and most of our examined patients had HPV infection. Thus, further examination of the correlation between HPV infection and FGFR2 expression, including whether HPV induces FGFR2 expression in cervical cancer, is necessary (15).

FGFR2 IIIc is widely expressed in various histological types of cancer cells, including adenocarcinoma, SCC and transitional cell carcinoma. Most studies indicated that FGFR2 IIIc expression is correlated with the aggressive growth of cancer cells $(38,39)$. In cervical cancer cases, FGFR2 IIIc mRNA was strongly expressed in the area of cancer cell infiltration in cancer tissues. The higher expression of FGFR2 IIIc in most cervical cancer cells and the strong expression in invasive fronts suggest that FGFR2 IIIc plays important roles in cancer cell growth. To the best of our knowledge, this is the first report on FGFR2 IIIc expression in cervical cancer.

To clarify whether FGFR2 IIIc contributes to the growth of cervical cancer cells, we attempted to prepare stable FGFR2 IIIc-transfected cancer cells. We were not able to successfully transfect the FGFR2 IIIc-expression vectors to ME-180 cells, but FGFR2 IIIc transfected CaSki cells expressed high levels of FGFR2 IIIc mRNA, and the cells 
rapidly grew, both in vitro and in vivo. Single-cell movement assay was performed to determine whether FGFR2 IIIc correlates with cervical cancer cell migration, but no significant difference between FGFR2 IIIc-transfected and Mock cells was found. These findings indicate that FGFR2 IIIc directly correlates with cervical cancer cell growth, but not with cell infiltration. Further studies on the inhibition of FGFR2 IIIc expression in cancer cells, including the use of small interfering RNA (siRNA), short hairpin RNA (shRNA) and monoclonal anti-FGFR2 IIIc antibodies, are necessary to determine the growth regulation of cervical cancer. Previously, a close relationship between FGFR2 IIIc expression and epithelial-mesenchymal transition (EMT) was reported (40). In this study, no stable FGFR2 IIIc-transfected CaSki cells showed spindle-like appearance. Further studies including the use of EMT markers in cells are necessary.

In summary, FGFR2 IIIc is expressed in the dysplastic or neoplastic cells in CIN patients, and all the cervical cancer patients clearly expressed high levels of FGFR2 IIIc and this expression induced cervical cancer cell growth. FGFR2 IIIc may be a new molecular target for cervical cancer therapy.

\section{Acknowledgements}

We express our appreciation to Mr. Kiyoshi Teduka and Takenori Fujii and Ms. Yoko Kawamoto, Taeko Suzuki and Kiyoko Kawahara (Department of Pathology, Nippon Medical School) for their excellent technical assistance. We also thank Dr Shin-Ichi Tsuchiya (Division of Surgical Pathology, Nippon Medical School Hospital) for preparing tissue blocks and Ms. Yuko Ono for preparing the manuscript. This study was supported by a Grant-in-Aid for Young Scientists from the Japan Society for the Promotion of Science to Y. Matsuda (B, No. 20790976).

\section{References}

1. Pectasides D, Kamposioras K, Papaxoinis G and Pectasides E: Chemotherapy for recurrent cervical cancer. Cancer Treat Rev 34: 603-613, 2008.

2. Jemal A, Siegel R, Ward E, et al: Cancer statistics, 2008. CA Cancer J Clin 58: 71-96, 2008.

3. Walboomers JM, Jacobs MV, Manos MM, et al: Human papillomavirus is a necessary cause of invasive cervical cancer worldwide. J Pathol 189: 12-19, 1999.

4. Wong AS, Leung PC and Auersperg N: Hepatocyte growth factor promotes in vitro scattering and morphogenesis of human cervical carcinoma cells. Gynecol Oncol 78: 158-165, 2000.

5. Kurban G, Ishiwata T, Kudo M, Yokoyama M, Sugisaki Y and Naito Z: Expression of keratinocyte growth factor receptor (KGFR/FGFR2 IIIb) in human uterine cervical cancer. Oncol Rep 11: 987-991, 2004.

6. Van Trappen PO, Steele D, Lowe DG, et al: Expression of vascular endothelial growth factor (VEGF)-C and VEGF-D, and their receptor VEGFR-3, during different stages of cervical carcinogenesis. J Pathol 201: 544-554, 2003.

7. Kuramoto H, Hongo A, Liu YX, et al: Immunohistochemical evaluation of insulin-like growth factor I receptor status in cervical cancer specimens. Acta Med Okayama 62: 251-259, 2008.

8. Dai H, Holm R, Kristensen GB, Abeler VM, Borresen-Dale AL and Helland A: Fibroblast growth factor receptor 3 (FGFR3) analyses of the S249C mutation and protein expression in primary cervical carcinomas. Anal Cell Pathol 23: 45-49, 2001.

9. Mayer TJ, Frauenhoffer EE and Meyers AC: Expression of epidermal growth factor and platelet-derived growth factor receptors during cervical carcinogenesis. In Vitro Cell Dev Biol Anim 36: 667-676, 2000.
10. Kuemmel S, Thomas A, Landt S, et al: Circulating vascular endothelial growth factors and their soluble receptors in preinvasive, invasive and recurrent cervical cancer. Anticancer Res 29: 641-645, 2009.

11. Mathur SP, Mathur RS, Gray EA, et al: Serum vascular endothelial growth factor C (VEGF-C) as a specific biomarker for advanced cervical cancer: relationship to insulin-like growth factor II (IGF-II), IGF binding protein 3 (IGF-BP3) and VEGF-A. Gynecol Oncol 98: 467-483, 2005.

12. Dobbs SP, Brown LJ, Ireland D, et al: Platelet-derived endothelial cell growth factor expression and angiogenesis in cervical intraepithelial neoplasia and squamous cell carcinoma of the cervix. Ann Diagn Pathol 4: 286-292, 2000.

13. Fujimoto J, Ichigo S, Hori M, Hirose R, Sakaguchi $\mathrm{H}$ and Tamaya T: Expression of basic fibroblast growth factor and its mRNA in advanced uterine cervical cancers. Cancer Lett 111: 21-26, 1997.

14. Hagemann T, Bozanovic T, Hooper S, et al: Molecular profiling of cervical cancer progression. Br J Cancer 96: 321-328, 2007.

15. Mathur RS and Mathur SP: Vascular endothelial growth factor (VEGF) up-regulates epidermal growth factor receptor (EGF-R) in cervical cancer in vitro: this action is mediated through HPV-E6 in HPV-positive cancers. Gynecol Oncol 97: 206-213, 2005.

16. Mitsuhashi A, Suzuka K, Yamazawa K, Matsui H, Seki K and Sekiya S: Serum vascular endothelial growth factor (VEGF) and VEGF-C levels as tumor markers in patients with cervical carcinoma. Cancer 103: 724-730, 2005.

17. Yang YC, Wang KL, Su TH, et al: Concurrent cisplatin-based chemoradiation for cervical carcinoma: tumor response, toxicity and serum cytokine profiles. Cancer Invest 24: 390-395, 2006.

18. Lebrecht A, Ludwig E, Huber A, et al: Serum vascular endothelial growth factor and serum leptin in patients with cervical cancer. Gynecol Oncol 85: 32-35, 2002.

19. Itoh N and Ornitz DM: Evolution of the Fgf and Fgfr gene families. Trends Genet 20: 563-569, 2004.

20. Jaye M, Schlessinger J and Dionne CA: Fibroblast growth factor receptor tyrosine kinases: molecular analysis and signal transduction. Biochim Biophys Acta 1135: 185-199, 1992.

21. Mohammadi M, Olsen SK and Ibrahimi OA: Structural basis for fibroblast growth factor receptor activation. Cytokine Growth Factor Rev 16: 107-137, 2005.

22. Eswarakumar VP, Lax I and Schlessinger J: Cellular signaling by fibroblast growth factor receptors. Cytokine Growth Factor Rev 16: 139-149, 2005.

23. Miki T, Bottaro DP, Fleming TP, et al: Determination of ligandbinding specificity by alternative splicing: two distinct growth factor receptors encoded by a single gene. Proc Natl Acad Sci USA 89: 246-250, 1992.

24. Oltean S, Sorg BS, Albrecht T, et al: Alternative inclusion of fibroblast growth factor receptor 2 exon IIIc in Dunning prostate tumors reveals unexpected epithelial mesenchymal plasticity. Proc Natl Acad Sci USA 103: 14116-14121, 2006.

25. Yan G, Fukabori Y, McBride G, Nikolaropolous S and McKeehan WL: Exon switching and activation of stromal and embryonic fibroblast growth factor (FGF)-FGF receptor genes in prostate epithelial cells accompany stromal independence and malignancy. Mol Cell Biol 13: 4513-4522, 1993.

26. Dutt A, Salvesen HB, Chen TH, et al: Drug-sensitive FGFR2 mutations in endometrial carcinoma. Proc Natl Acad Sci USA 105: 8713-8717, 2008.

27. Katoh Y and Katoh M: FGFR2-related pathogenesis and FGFR2-targeted therapeutics (Review). Int J Mol Med 23: 307-311, 2009.

28. Katoh M: Cancer genomics and genetics of FGFR2 (Review). Int J Oncol 33: 233-237, 2008.

29. Hudelist G, Czerwenka K, Singer C, Pischinger K, Kubista E and Manavi M: cDNA array analysis of cytobrush-collected normal and malignant cervical epithelial cells: a feasibility study. Cancer Genet Cytogenet 158: 35-42, 2005.

30. Chopra V, Dinh TV and Hannigan EV: Circulating serum levels of cytokines and angiogenic factors in patients with cervical cancer. Cancer Invest 16: 152-159, 1998.

31. Cho K, Ishiwata T, Uchida E, et al: Enhanced expression of keratinocyte growth factor and its receptor correlates with venous invasion in pancreatic cancer. Am J Pathol 170: 1964-1974, 2007.

32. Ishiwata T: Immunohistochemical and in situ hybridization analysis of lumican in colorectal carcinoma. In: Immunohistochemistry and In Situ Hybridization of Human Carcinomas. Ma H (ed). Elsevier Academic Press, Burlington, pp237-243, 2005. 
33. Ishiwata T, Friess H, Buchler MW, Lopez ME and Korc M: Characterization of keratinocyte growth factor and receptor expression in human pancreatic cancer. Am J Pathol 153: 213-222, 1998

34. Kawamoto M, Ishiwata $\mathrm{T}$, Cho $\mathrm{K}$, et al: Nestin expression correlates with nerve and retroperitoneal tissue invasion in pancreatic cancer. Hum Pathol 40: 189-198, 2009.

35. Naito Z, Ishiwata T, Kurban G, et al: Expression and accumulation of lumican protein in uterine cervical cancer cells at the periphery of cancer nests. Int J Oncol 20: 943-948, 2002.

36. Hammes LS, Tekmal RR, Naud P, et al: Up-regulation of VEGF, c-fms and COX-2 expression correlates with severity of cervical cancer precursor (CIN) lesions and invasive disease. Gynecol Oncol 110: 445-451, 2008.
37. Soufla G, Sifakis S, Baritaki S, Zafiropoulos A, Koumantakis E and Spandidos DA: VEGF, FGF2, TGFB1 and TGFBR1 mRNA expression levels correlate with the malignant transformation of the uterine cervix. Cancer Lett 221: 105-118, 2005.

38. Kwabi-Addo B, Ropiquet F, Giri D and Ittmann M: Alternative splicing of fibroblast growth factor receptors in human prostate cancer. Prostate 46: 163-172, 2001.

39. Cha JY, Lambert QT, Reuther GW and Der CJ: Involvement of fibroblast growth factor receptor 2 isoform switching in mammary oncogenesis. Mol Cancer Res 6: 435-445, 2008.

40. Chaffer CL, Brennan JP, Slavin JL, Blick T, Thompson EW and Williams ED: Mesenchymal-to-epithelial transition facilitates bladder cancer metastasis: role of fibroblast growth factor receptor-2. Cancer Res 66: 11271-11278, 2006. 